\title{
Two-loop QED corrections to the Altarelli-Parisi splitting functions
}

\author{
Daniel de Florian, ${ }^{a}$ Germán F.R. Sborlini ${ }^{b}$ and Germán Rodrigo ${ }^{b}$ \\ ${ }^{a}$ International Center for Advanced Studies (ICAS), UNSAM, \\ Campus Miguelete, 25 de Mayo y Francia, (1650) Buenos Aires, Argentina \\ ${ }^{b}$ Instituto de Física Corpuscular, Universitat de València, \\ Consejo Superior de Investigaciones Cientificas, \\ Parc Cientific, E-46980 Paterna, Valencia, Spain \\ E-mail: deflo@unsam.edu.ar, german.sborlini@ific.uv.es, \\ german.rodrigo@csic.es
}

Abstract: We compute the two-loop QED corrections to the Altarelli-Parisi (AP) splitting functions by using a deconstructive algorithmic Abelianization of the well-known NLO QCD corrections. We present explicit results for the full set of splitting kernels in a basis that includes the leptonic distribution functions that, starting from this order in the QED coupling, couple to the partonic densities. Finally, we perform a phenomenological analysis of the impact of these corrections in the splitting functions.

KEYWORDS: NLO Computations, QCD Phenomenology

ARXIV EPRINT: 1606.02887 


\section{Contents}

1 Introduction 1

2 Evolution equations and definitions 2

$\begin{array}{ll}2.1 \text { Constraints from sum rules } & 6\end{array}$

$\begin{array}{lll}3 & \text { Splitting kernels at } \mathcal{O}\left(\alpha^{2}\right) & 7\end{array}$

4 Phenomenological impact of QED corrections 10

5 Conclusions 12

\section{Introduction}

The availability of highly precise experimental data collected in the LHC Run II demands to push forward the accuracy frontier at the theoretical side. In the context of perturbative QCD, higher-order corrections have been computed for a large variety of processes, reaching even $\mathrm{N}^{3} \mathrm{LO}$ accuracy in some cases. In consequence, some contributions, that were considered sub-leading long time ago, are starting to compete with QCD effects and might be crucial to compare theoretical predictions with experimental data.

Besides the substantial work accomplished in the perturbative sector, it is essential to reach the same level of accuracy on the non-perturbative side, i.e., on the parton distribution functions (PDFs). The calculation of the NNLO corrections to the splitting functions performed in [1-4] and the development of modern parton distribution analysis [5-10] allows to achieve the required precision in QCD.

Since $\alpha_{\mathrm{S}}^{2} \sim \alpha$, NLO ElectroWeak (EW) corrections compete with NNLO QCD contributions. As a result, an accurate description for many observables requires the inclusion of the corresponding EW effects, which might account for a few percent level correction. Recent work has been performed on the PDF sector to incorporate the EW effect (the dominant QED terms) in the evolution equations [11-13]. The appearance of the photon and the leptonic densities is the first main modification in the evolution of PDFs due to the inclusion of QED corrections. The EW corrections to PDFs need to be carefully studied for precise predictions at the LHC, as concluded from modern analysis performed up to NNLO in QCD and LO in QED. In fact, it was shown that the corrections induced are non negligible and, moreover, become crucial at higher energies [14-17].

Heretofore, the evolution of parton densities was performed using only LO QED kernels. Recently, we presented the calculation of the NLO combined QCD-QED contributions (i.e., $\mathcal{O}\left(\alpha \alpha_{\mathrm{S}}\right)$ ) to the evolution kernels [18]. Also, one-loop corrections to double [19-22] and triple [23-25] collinear splitting functions with photons have been computed. With 
the improvement of accuracy as the main motivation, we present for the first time, the expressions for the Altarelli-Parisi (AP) splitting functions [26] to $\mathcal{O}\left(\alpha^{2}\right)$ that completes the full set of two-loop kernels. Following the algorithmic procedure developed in ref. [18], we make use of the diagram-by-diagram classification available in the original NLO QCD results presented in refs. [27-29] and, then, we modified consistently their color structure to account for the gluon-photon replacement. In this case, we explicitly concentrate on the QED corrections, without including those arising from Weak bosons, which only become relevant for very extreme kinematical conditions, where their masses are neglected in comparison to other scales involved in the process.

The structure of the paper is as follows. In section 2 we recall the evolution equations for the different distributions and the corresponding kernels, introducing the notation required to present our results. Also, we present there the constraints from sum rules that determine the behaviour of splitting kernels in the end-point region (i.e. $x=1$ ). In section 3 we summarize the algorithm that we use to obtain the QED corrections to the splitting functions and present the corresponding kernels. Using these formulae, we study the changes introduced in the AP kernels by both $\mathcal{O}\left(\alpha^{2}\right)$ and $\mathcal{O}\left(\alpha \alpha_{\mathrm{S}}\right)$, in section 4. Finally, conclusions are given in section 5 .

\section{Evolution equations and definitions}

In the context of combined QCD-QED contributions, it is mandatory to take into account lepton distributions. In ref. [18], we computed the $\mathcal{O}\left(\alpha \alpha_{\mathrm{S}}\right)$ contributions to the AP kernels and we showed that leptons decouple from the QCD sector at that accuracy. Thus, in that case, we neglected lepton distributions. Moreover, this simplification remains true for $\mathcal{O}\left(\alpha \alpha_{\mathrm{S}}^{n}\right)$ because the quark-lepton mixing appears starting at $\mathcal{O}\left(\alpha^{2}\right)$. Therefore, here we follow the path established in ref. [30] and obtain the exact set of evolution equations for the combined QCD-QED model in a proper basis.

As usual, the first step consists in writing down the evolution equations for quark, lepton, gluon and photon distributions. These equations are obtained starting from those available in ref. [18] by adding lepton distributions and the corresponding AP kernels, $P_{i j}$. Using the standard definition for the convolution operator, i.e.

$$
(f \otimes g)(x)=\int_{x}^{1} \frac{d y}{y} f\left(\frac{x}{y}\right) g(y)
$$

and introducing $t=\ln \left(\mu^{2}\right)$ as the evolution variable, we have

$$
\begin{aligned}
& \frac{d g}{d t}=\sum_{f} P_{g f} \otimes f+\sum_{f} P_{g \bar{f}} \otimes \bar{f}+P_{g g} \otimes g+P_{g \gamma} \otimes \gamma, \\
& \frac{d \gamma}{d t}=\sum_{f} P_{\gamma f} \otimes f+\sum_{f} P_{\gamma \bar{f}} \otimes \bar{f}+P_{\gamma g} \otimes g+P_{\gamma \gamma} \otimes \gamma,
\end{aligned}
$$




$$
\begin{aligned}
& \frac{d q_{i}}{d t}=\sum_{f} P_{q_{i} f} \otimes f+\sum_{f} P_{q_{i} \bar{f}} \otimes \bar{f}+P_{q_{i} g} \otimes g+P_{q_{i} \gamma} \otimes \gamma \\
& \frac{d l_{i}}{d t}=\sum_{f} P_{l_{i} f} \otimes f+\sum_{f} P_{l_{i} \bar{f}} \otimes \bar{f}+P_{l_{i} g} \otimes g+P_{l_{i} \gamma} \otimes \gamma
\end{aligned}
$$

and similarly for antiparticles by using charge conjugation invariance. Here the sum over fermions $f$ runs over all the active flavours of quarks $\left(n_{F}\right)$ and leptons $\left(n_{L}\right)$. In the previous formulae, $\mu$ represents the factorization scale.

Along this work we will present the expressions for the splitting functions including QCD and QED corrections. Thus, we expand them according to

$$
P_{i j}=a_{\mathrm{S}} P_{i j}^{(1,0)}+a P_{i j}^{(0,1)}+a_{\mathrm{S}}^{2} P_{i j}^{(2,0)}+a_{\mathrm{S}} a P_{i j}^{(1,1)}+a^{2} P_{i j}^{(0,2)}+\ldots,
$$

where the upper indices indicate the (QCD,QED) order of the calculation, while

$$
a_{\mathrm{S}} \equiv \frac{\alpha_{\mathrm{S}}}{2 \pi}, \quad a \equiv \frac{\alpha}{2 \pi},
$$

allow to set the standard normalization of the splitting functions.

The presence of electromagnetic interactions introduces a charge dependence in the splitting functions. Moreover, due to higher-order QED corrections, a mixing among leptons and QCD partons might take place, which leads to more complicated evolution equations. In fact, in the most general case, eqs. (2.2)-(2.5) constitute a system of $20 \times 20$ coupled first-order differential equations. However, notable simplifications are achieved at each order of the truncated expansion by imposing physical constraints. For instance, at $\mathcal{O}\left(\alpha \alpha_{\mathrm{S}}^{n}\right)$, the kernels depend on the electric charge of the initiating fermions (up or down), such that in general $P_{q}^{(1, n)} \sim e_{q}^{2}$. As we will show later, at $\mathcal{O}\left(\alpha^{2}\right)$, the charge content of the $P_{q l}$ kernels becomes non trivial due to the exchange of a pair of photons.

The quark splitting functions are decomposed as

$$
\begin{aligned}
P_{q_{i} q_{k}} & =\delta_{i k} P_{q q}^{V}+P_{q q}^{S}, \\
P_{q_{i} \bar{q}_{k}} & =\delta_{i k} P_{q \bar{q}}^{V}+P_{q \bar{q}}^{S}, \\
P_{q}^{ \pm} & =P_{q q}^{V} \pm P_{q \bar{q}}^{V},
\end{aligned}
$$

which act as a definition of $P_{q q}^{V}$ and $P_{q \bar{q}}^{V}$, i.e. the non-singlet components. In a completely analogous way, we write

$$
\begin{aligned}
P_{l_{i} l_{k}} & =\delta_{i k} P_{l l}^{V}+P_{l l}^{S}, \\
P_{l_{i} \bar{l}_{k}} & =\delta_{i k} P_{l \bar{l}}^{V}+P_{l \bar{l}}^{S}, \\
P_{l}^{ \pm} & =P_{l l}^{V} \pm P_{l \bar{l}}^{V},
\end{aligned}
$$

for the lepton kernels. For mixed lepton-quark splittings we use $P_{l q}^{S} \equiv P_{l q}$ to simplify the notation.

The canonical basis of distributions is given by

$$
\mathcal{B}_{\mathrm{c}}=\{u, \bar{u}, \ldots, t, \bar{t}, e, \bar{e}, \ldots, \tau, \bar{\tau}, g, \gamma\},
$$


when considering the full charged-fermion content of the Standard Model. Working with a reduced amount of fermions simply involves removing them from the previous basis. As explained in ref. [30], $\mathcal{B}_{\mathrm{c}}$ is not the optimal choice to reduce the mixing among the different parton distributions in the evolution system. Thus, for $n_{F}=5$ active flavours it is more suitable to work with the following set:

$$
\mathcal{B}=\left\{u_{v}, d_{v}, s_{v}, c_{v}, b_{v}, e_{v}, \mu_{v}, \tau_{v}, \Delta_{u c}, \Delta_{d s}, \Delta_{s b}, \Delta_{2}^{l}, \Delta_{U D}, \Delta_{3}^{l}, \Sigma, \Sigma^{l}, g, \gamma\right\}
$$

where

$$
\begin{aligned}
q_{v} & =q_{i}-\bar{q}_{i}, \\
l_{v} & =l_{i}-\bar{l}_{i},
\end{aligned}
$$

are the valence distributions, whilst

$$
\begin{aligned}
\Delta_{u c} & =u+\bar{u}-c-\bar{c} \\
\Delta_{d s} & =d+\bar{d}-s-\bar{s} \\
\Delta_{s b} & =s+\bar{s}-b-\bar{b} \\
\Delta_{2}^{l} & =e+\bar{e}-\mu-\bar{\mu} \\
\Delta_{U D} & =u+\bar{u}+c+\bar{c}-d-\bar{d}-s-\bar{s}-b-\bar{b}, \\
\Delta_{3}^{l} & =e+\bar{e}+\mu+\bar{\mu}-2(\tau+\bar{\tau}), \\
\Sigma & =\sum_{i=1}^{n_{F}}\left(q_{i}+\bar{q}_{i}\right), \\
\Sigma^{l} & =\sum_{i=1}^{n_{L}}\left(l_{i}+\bar{l}_{i}\right),
\end{aligned}
$$

are the remaining combinations (gluons and photons are treated separately). To include the top distribution in case of a six flavour analysis, it is necessary to introduce the elements $\left\{t_{v}, \Delta_{c t}\right\}$ and to extend the definitions of $\Delta_{U D}$ and $\Sigma$.

As we mentioned before, QED interactions introduce a classification of the different fermions according to the absolute value of their electromagnetic (EM) charges. Thus, there are three possible fermionic sectors: up-like quarks ( $u$ and $\left.e_{u}=2 / 3\right)$, down-like quarks $(d$ and $\left.e_{d}=1 / 3\right)$ and leptons $\left(l\right.$ with $\left.e_{l}=1\right)$. Particles inside each sector are indistinguishable by QCD-QED interactions. Also, it is useful to define

$$
\begin{aligned}
\Delta P_{f F}^{S} & \equiv P_{f F}^{S}-P_{f \bar{F}}^{S}, \\
\bar{P}_{f F}^{S} & \equiv P_{f F}^{S}+P_{f \bar{F}}^{S},
\end{aligned}
$$

where $f$ and $F$ denote the possible fermion subgroups ( $u, d$ or $l$ ). Notice that in the context of QCD-QED, it might occur that $P_{l q} \neq P_{q l}$ due to higher-order contributions. However, at $\mathcal{O}\left(\alpha^{2}\right)$, they are the same and the equality can be used to achieve further simplifications. Moreover, at this order, it is verified that

$$
\Delta P_{f F}^{S} \equiv 0,
$$

due to charge conjugation invariance. 
In the most general case, the corresponding QCD-QED combined evolution equations for the distributions in the optimized basis are given by:

$$
\begin{aligned}
\frac{d q_{v_{i}}}{d t} & =P_{q_{i}}^{-} \otimes q_{v_{i}}+\sum_{j=1}^{n_{F}} \Delta P_{q_{i} q_{j}}^{S} \otimes q_{v_{j}}+\Delta P_{q_{i} l}^{S} \otimes\left(\sum_{j=1}^{n_{L}} l_{v_{j}}\right), \\
\frac{d l_{v_{i}}}{d t} & =P_{l}^{-} \otimes l_{v_{i}}+\sum_{j=1}^{n_{F}} \Delta P_{l q_{j}}^{S} \otimes q_{v_{j}}+\Delta P_{l l}^{S} \otimes\left(\sum_{j=1}^{n_{L}} l_{v_{j}}\right),
\end{aligned}
$$

for valence distributions,

$$
\begin{aligned}
\frac{d \Sigma}{d t}= & \frac{P_{u}^{+}+P_{d}^{+}}{2} \otimes \Sigma+\frac{P_{u}^{+}-P_{d}^{+}}{2} \otimes \Delta_{U D}+\frac{n_{u} \bar{P}_{u u}^{S}+n_{d} \bar{P}_{d d}^{S}+\left(n_{u}+n_{d}\right) \bar{P}_{u d}^{S}}{2} \otimes \Sigma \\
& +\frac{n_{u} \bar{P}_{u u}^{S}-n_{d} \bar{P}_{d d}^{S}-\left(n_{u}-n_{d}\right) \bar{P}_{u d}^{S}}{2} \otimes \Delta_{U D}+\left(n_{u} \bar{P}_{u l}^{S}+n_{d} \bar{P}_{d l}^{S}\right) \otimes \Sigma^{l} \\
& +2\left(n_{u} P_{u g}+n_{d} P_{d g}\right) \otimes g+2\left(n_{u} P_{u \gamma}+n_{d} P_{d \gamma}\right) \otimes \gamma \\
\frac{d \Sigma^{l}}{d t}= & n_{L} \frac{\bar{P}_{l u}^{S}+\bar{P}_{l d}^{S}}{2} \otimes \Sigma+n_{L} \frac{\bar{P}_{l u}^{S}-\bar{P}_{l d}^{S}}{2} \otimes \Delta_{U D}+\left(P_{l}^{+}+n_{L} \bar{P}_{l l}^{S}\right) \otimes \Sigma^{l} \\
& +2 n_{L}\left(P_{l g} \otimes g+P_{l \gamma} \otimes \gamma\right)
\end{aligned}
$$

for the singlets and

$$
\begin{aligned}
\frac{d\left\{\Delta_{u c}, \Delta_{c t}\right\}}{d t}= & P_{u}^{+} \otimes\left\{\Delta_{u c}, \Delta_{c t}\right\}, \\
\frac{d\left\{\Delta_{d s}, \Delta_{s b}\right\}}{d t}= & P_{d}^{+} \otimes\left\{\Delta_{d s}, \Delta_{s b}\right\} \\
\frac{d \Delta_{2}^{l}}{d t}= & P_{l}^{+} \otimes \Delta_{2}^{l}, \\
\frac{d \Delta_{U D}}{d t}= & \frac{P_{u}^{+}+P_{d}^{+}}{2} \otimes \Delta_{U D}+\frac{P_{u}^{+}-P_{d}^{+}}{2} \otimes \Sigma+\frac{n_{u} \bar{P}_{u u}^{S}-n_{d} \bar{P}_{d d}^{S}+\left(n_{u}-n_{d}\right) \bar{P}_{u d}^{S}}{2} \otimes \Sigma \\
& +\frac{n_{u} \bar{P}_{u u}^{S}+n_{d} \bar{P}_{d d}^{S}-\left(n_{u}+n_{d}\right) \bar{P}_{u d}^{S}}{2} \otimes \Delta_{U D}+\left(n_{u} \bar{P}_{u l}^{S}-n_{d} \bar{P}_{d l}^{S}\right) \otimes \Sigma^{l} \\
& +2\left(n_{u} P_{u g}-n_{d} P_{d g}\right) \otimes g+2\left(n_{u} P_{u \gamma}-n_{d} P_{d \gamma}\right) \otimes \gamma, \\
\frac{d \Delta_{3}^{l}}{d t}= & P_{l}^{+} \otimes \Delta_{3}^{l},
\end{aligned}
$$

for the remaining fermionic distributions. Here $n_{u}\left(n_{d}\right)$ refers to the active number of $u(d)$ type-quarks, respectively $\left(n_{F}=n_{u}+n_{d}\right)$, and $n_{L}$ is the number of leptons under consideration. It is worth noticing that only $\Delta_{i j}$ and $\Delta_{i}^{l}$ decouple from the other distributions. Besides that, if we restrict to $\mathcal{O}\left(\alpha \alpha_{\mathrm{S}}\right)$, we recover the equations presented in ref. [18]. At $\mathcal{O}\left(\alpha^{2}\right)$, the splitting kernels are charge dependent but $\Delta P^{S} \equiv 0$. Thus, in that case, eqs. (2.29) and (2.30) become

$$
\begin{aligned}
& \frac{d q_{v_{i}}}{d t}=P_{q_{i}}^{-} \otimes q_{v_{i}}, \\
& \frac{d l_{v_{i}}}{d t}=P_{l}^{-} \otimes l_{v_{i}},
\end{aligned}
$$


and

$$
\bar{P}_{i j}^{S} \equiv 2 P_{i j}^{S}, \quad P_{l q}^{S} \equiv P_{q l}^{S} .
$$

Moreover, if we only allow QED interactions, all splitting kernels with gluons vanish and the gluon distribution is decoupled from the other ones.

\subsection{Constraints from sum rules}

On one side, QCD-QED interactions preserve the fermion number. In particular, this implies that splitting kernels must fulfil

$$
\int_{0}^{1} d x P_{f}^{-}=0
$$

because the factorization scale $\mu$ is arbitrary. On the other hand, the arbitrariness of $\mu$ also implies that the momentum of the proton is conserved during the evolution. Using the parton model, we have

$$
0=\frac{d P}{d t}=\int_{0}^{1} d x x\left(\frac{d g}{d t}+\frac{d \gamma}{d t}+\sum_{f}\left(\frac{d f}{d t}+\frac{d \bar{f}}{d t}\right)\right),
$$

where the sum is over all the possible fermion flavours (both quarks and leptons). If we express eq. (2.42) by using the optimized basis, we impose its validity in each component. The non-trivial constraints are:

- Gluon and photon components,

$$
\begin{aligned}
& \int_{0}^{1} d x x\left(2 n_{d} P_{d g}+2 n_{u} P_{u g}+2 n_{L} P_{l g}+P_{\gamma g}+P_{g g}\right)=0, \\
& \int_{0}^{1} d x x\left(2 n_{d} P_{d \gamma}+2 n_{u} P_{u \gamma}+2 n_{L} P_{l \gamma}+P_{g \gamma}+P_{\gamma \gamma}\right)=0 ;
\end{aligned}
$$

- $\Delta_{U D}$ component,

$$
\begin{aligned}
\int_{0}^{1} d x x & \left(\frac{P_{u}^{+}-P_{d}^{+}}{2}+n_{L} \frac{\bar{P}_{l u}^{S}-\bar{P}_{l d}^{S}}{2}+\frac{n_{u} \bar{P}_{u u}^{S}-n_{d} \bar{P}_{d d}^{S}}{2}-\frac{\left(n_{u}-n_{d}\right) \bar{P}_{u d}^{S}}{2}\right. \\
& \left.+\frac{P_{g u}-P_{g d}}{2}+\frac{P_{\gamma u}-P_{\gamma d}}{2}\right)=0
\end{aligned}
$$

- and, finally, the singlet components ( $\Sigma$ and $\Sigma^{l}$, respectively),

$$
\begin{aligned}
& \int_{0}^{1} d x x\left(\frac{P_{u}^{+}+P_{d}^{+}}{2}+n_{L} \frac{\bar{P}_{l u}^{S}+\bar{P}_{l d}^{S}}{2}+\frac{n_{u} \bar{P}_{u u}^{S}+n_{d} \bar{P}_{d d}^{S}}{2}+\frac{n_{F} \bar{P}_{u d}^{S}}{2}\right. \\
&\left.+\frac{P_{g u}+P_{g d}}{2}+\frac{P_{\gamma u}+P_{\gamma d}}{2}\right)=0, \\
& \int_{0}^{1} d x x \quad\left(n_{u} \bar{P}_{u l}^{S}+n_{d} \bar{P}_{d l}^{S}+n_{L} \bar{P}_{l l}^{S}+P_{l}^{+}+P_{g l}+P_{\gamma l}\right)=0 .
\end{aligned}
$$

In the following sections, we will use these equations to provide a strict check of the calculation and, at the same time, fix the value of the splitting kernels in the end-point $x=1$. 


\section{$3 \quad$ Splitting kernels at $\mathcal{O}\left(\alpha^{2}\right)$}

Let's start by recalling some well-known results for the lowest order splitting functions. At $\mathcal{O}\left(\alpha_{\mathrm{S}}\right)$, only QCD partons are involved [26]; thus,

$$
\begin{aligned}
& P_{q q}^{(1,0)}(x)=C_{F}\left[\frac{1+x^{2}}{(1-x)_{+}}+\frac{3}{2} \delta(1-x)\right]=C_{F}\left[p_{q q}(x)+\frac{3}{2} \delta(1-x)\right], \\
& P_{q g}^{(1,0)}(x)=T_{R}\left[x^{2}+(1-x)^{2}\right]=T_{R} p_{q g}(x), \\
& P_{g q}^{(1,0)}(x)=C_{F}\left[\frac{1+(1-x)^{2}}{x}\right]=C_{F} p_{g q}(x), \\
& P_{g g}^{(1,0)}(x)=2 C_{A}\left[\frac{x}{(1-x)_{+}}+\frac{1-x}{x}+x(1-x)\right]+\frac{\beta_{0}}{2} \delta(1-x),
\end{aligned}
$$

with $\beta_{0}=\frac{11 N_{C}-4 n_{F} T_{R}}{3}$ and the plus distribution defined as

$$
\int_{0}^{1} d x \frac{f(x)}{(1-x)_{+}}=\int_{0}^{1} d x \frac{f(x)-f(1)}{1-x},
$$

for any regular test function $f$. As usual, the normalization of the fundamental representation is set to $T_{R}=1 / 2$ and

$$
C_{A}=N_{C}, \quad C_{F}=\frac{N_{C}^{2}-1}{2 N_{C}},
$$

are the $\mathrm{SU}\left(N_{C}\right)$ group factors. In particular, for $\mathrm{QCD}\left(N_{C}=3\right)$, we have $C_{A}=3$ and $C_{F}=4 / 3$. Notice that these expressions provide a definition for the color-stripped splitting functions $p_{i j}$, which will be used to simplify the presentation of higher-order corrections. At $\mathcal{O}(\alpha)$, splitting processes can be described by replacing the color factors in $P_{i j}^{(1,0)}$ with the corresponding EM charges. In this way, we have [30]

$$
\begin{aligned}
P_{f f}^{(0,1)}(x) & =e_{f}^{2}\left[p_{q q}(x)+\frac{3}{2} \delta(1-x)\right], \\
P_{f \gamma}^{(0,1)}(x) & =e_{f}^{2} p_{q g}(x) \\
P_{\gamma f}^{(0,1)}(x) & =e_{f}^{2} p_{g q}(x), \\
P_{\gamma \gamma}^{(0,1)}(x) & =-\frac{2}{3} \sum_{f} e_{f}^{2} \delta(1-x),
\end{aligned}
$$

where $f$ denotes any fermion (quark or lepton) with its corresponding EM charge $e_{f}$, and

$$
\sum_{f} e_{f}^{a}=N_{C} \sum_{j=1}^{n_{F}} e_{q_{j}}^{a}+\sum_{j=1}^{n_{L}} e_{l_{j}}^{a},
$$

is the sum over fermion charges, taking into account that quark-photon interactions are degenerate due to color degrees of freedom $\left(N_{C}\right)$.

In order to obtain the pure two-loop QED corrections $P_{i j}^{(0,2)}$, we follow the ideas depicted in ref. [18]. We start from the results on the two-loop QCD anomalous dimensions 
in the light-cone gauge, originally performed for the non-singlet component by Curci, Furmanski and Petronzio in ref. [27] and extended to the singlet case in refs. [28, 29]. ${ }^{1}$ Then we take the corresponding Abelian limit, which involves replacing each gluon by a photon [32]. This automatically avoids the presence of diagrams with non-Abelian vertices (at least in pure QED). The last step consists in replacing the original color structure with the one obtained after the double replacement $g \rightarrow \gamma$, and multiplying by the EM charge of the fermions involved in the process.

However, as anticipated in section 2, lepton distributions enter in the evolution of the system at $\mathcal{O}\left(\alpha^{2}\right)$, which forces us to compute also lepton-quark and lepton-photon kernels at this order. The procedure is completely analogous to the one described before.

There is a subtlety related with the presence of quark loops in pure QCD results. In that case, gluons couple in the same way to all quark flavours, originating a factor $n_{F}$. Once we replace gluons with photons, virtual leptons are also allowed inside the loop. Both for leptons and quarks, the QED coupling is proportional to their EM charges. In consequence, the replacement

$$
n_{F} \rightarrow \sum_{f} e_{f}^{2}
$$

has to be implemented in all the contributions arising from quark loops in the pure QCD kernels.

Another subtle point that we must carefully treat is the presence of massive EW bosons. As we mentioned before, we neglect their contribution in this work. This is due to the fact that their mass is kept strictly non-vanishing, thus acting as an IR-regulator. In other terms, IR-singular diagrams for processes involving heavy EW bosons can always be treated by making use of QCD-QED splitting functions and factorizing the massive particle into the hard scattering subprocess.

So, let's present the explicit results. In first place, kernels involving gluons vanish at this order; hence,

$$
\begin{array}{lll}
P_{f g}^{(0,2)}=0, & P_{g f}^{(0,2)}=0, & P_{\gamma g}^{(0,2)}=0, \\
P_{g \gamma}^{(0,2)}=0, & P_{g g}^{(0,2)}=0 . &
\end{array}
$$

Then, we consider those kernels which involve quarks and photons,

$$
\begin{aligned}
P_{q \gamma}^{(0,2)}= & \frac{C_{A} e_{q}^{4}}{2}\left\{4-9 x-(1-4 x) \ln (x)-(1-2 x) \ln ^{2}(x)+4 \ln (1-x)\right. \\
& \left.+p_{q g}(x)\left[2 \ln ^{2}\left(\frac{1-x}{x}\right)-4 \ln \left(\frac{1-x}{x}\right)-\frac{2 \pi^{2}}{3}+10\right]\right\} \\
P_{\gamma q}^{(0,2)}= & e_{q}^{4}\left[-\left(3 \ln (1-x)+\ln ^{2}(1-x)\right) p_{g q}(x)+\left(2+\frac{7}{2} x\right) \ln (x)-\left(1-\frac{x}{2}\right) \ln ^{2}(x)\right. \\
& \left.-2 x \ln (1-x)-\frac{7}{2} x-\frac{5}{2}\right]-e_{q}^{2}\left(\sum_{f} e_{f}^{2}\right)\left[\frac{4}{3} x+p_{g q}(x)\left(\frac{20}{9}+\frac{4}{3} \ln (1-x)\right)\right],
\end{aligned}
$$

\footnotetext{
${ }^{1}$ For a complete review of the previous developments in the computation of higher-order corrections to the splitting kernels and the anomalous dimension, see ref. [31] and the references therein.
} 


$$
\begin{aligned}
P_{q q}^{V(0,2)}= & -e_{q}^{4}\left[\left(2 \ln (x) \ln (1-x)+\frac{3}{2} \ln (x)\right) p_{q q}(x)+\frac{3+7 x}{2} \ln (x)\right. \\
& \left.+\frac{1+x}{2} \ln ^{2}(x)+5(1-x)+\left(\frac{\pi^{2}}{2}-\frac{3}{8}-6 \zeta_{3}\right) \delta(1-x)\right] \\
& -e_{q}^{2}\left(\sum_{f} e_{f}^{2}\right)\left[\frac{4}{3}(1-x)+p_{q q}(x)\left(\frac{2}{3} \ln (x)+\frac{10}{9}\right)+\left(\frac{2 \pi^{2}}{9}+\frac{1}{6}\right) \delta(1-x)\right], \\
P_{q \bar{q}}^{V(0,2)}= & e_{q}^{4}\left[4(1-x)+2(1+x) \ln (x)+2 p_{q q}(-x) S_{2}(x)\right], \\
P_{q Q}^{S(0,2)}= & P_{q \bar{Q}}^{S(0,2)}=C_{A} e_{q}^{2} e_{Q}^{2} p_{s}(x),
\end{aligned}
$$

where $\{q, Q\}$ denote different quark flavours and we defined the function

$$
p_{s}(x)=\frac{20}{9 x}-2+6 x-\frac{56}{9} x^{2}+\left(1+5 x+\frac{8}{3} x^{2}\right) \ln (x)-(1+x) \ln ^{2}(x),
$$

which appears in all the higher-order corrections to the singlet components. The function $S_{2}(x)$ is given by $[28,33]$

$$
S_{2}(x)=\int_{\frac{x}{1+x}}^{\frac{1}{1+x}} \frac{d z}{z} \ln \left(\frac{1-z}{z}\right)=\frac{\ln ^{2}(x)}{2}-\zeta_{2}-2 \operatorname{Li}_{2}(-x)-2 \ln (x) \ln (1+x) .
$$

In these formulae, $\zeta_{n}$ is the Riemann zeta function, which verifies $\zeta_{2}=\pi^{2} / 6$ and $\zeta_{3} \approx$ 1.202057 .

In an analogous way, splitting functions with leptons and photons are given by

$$
\begin{aligned}
& P_{l \gamma}^{(0,2)}= \frac{e_{l}^{4}}{C_{A} e_{q}^{4}} P_{q \gamma}^{(0,2)} \\
& P_{\gamma l}^{(0,2)}= e_{l}^{4}\left[-\left(3 \ln (1-x)+\ln ^{2}(1-x)\right) p_{g q}(x)+\left(2+\frac{7}{2} x\right) \ln (x)-\left(1-\frac{x}{2}\right) \ln ^{2}(x)\right. \\
&\left.-2 x \ln (1-x)-\frac{7}{2} x-\frac{5}{2}\right]-e_{l}^{2}\left(\sum_{f} e_{f}^{2}\right)\left[\frac{4}{3} x+p_{g q}(x)\left(\frac{20}{9}+\frac{4}{3} \ln (1-x)\right)\right] \\
& P_{l l}^{V(0,2)}=-e_{l}^{4}\left[\left(2 \ln (x) \ln (1-x)+\frac{3}{2} \ln (x)\right) p_{q q}(x)+\frac{3+7 x}{2} \ln (x)\right. \\
&\left.+\frac{1+x}{2} \ln ^{2}(x)+5(1-x)+\left(\frac{\pi^{2}}{2}-\frac{3}{8}-6 \zeta_{3}\right) \delta(1-x)\right] \\
&-e_{l}^{2}\left(\sum_{f} e_{f}^{2}\right)\left[\frac{4}{3}(1-x)+p_{q q}(x)\left(\frac{2}{3} \ln (x)+\frac{10}{9}\right)+\left(\frac{2 \pi^{2}}{9}+\frac{1}{6}\right) \delta(1-x)\right] \\
& \frac{e_{l}^{4}}{e_{q}^{4}} P_{q \bar{q}}^{V(0,2)}, \\
& P_{l \bar{l}}^{V(0,2)} P_{l L}^{S(0,2)}= \\
& P_{l \bar{L}}^{S(0,2)}=e_{l}^{2} e_{L}^{2} p_{s}(x) .
\end{aligned}
$$

Mixed quark-lepton evolution kernels are given by

$$
\begin{aligned}
& P_{l q}^{S(0,2)}=P_{l \bar{q}}^{S(0,2)}=e_{l}^{2} e_{q}^{2} p_{s}(x), \\
& P_{q l}^{S(0,2)}=P_{q \bar{l}}^{S(0,2)}=C_{A} e_{l}^{2} e_{q}^{2} p_{s}(x),
\end{aligned}
$$




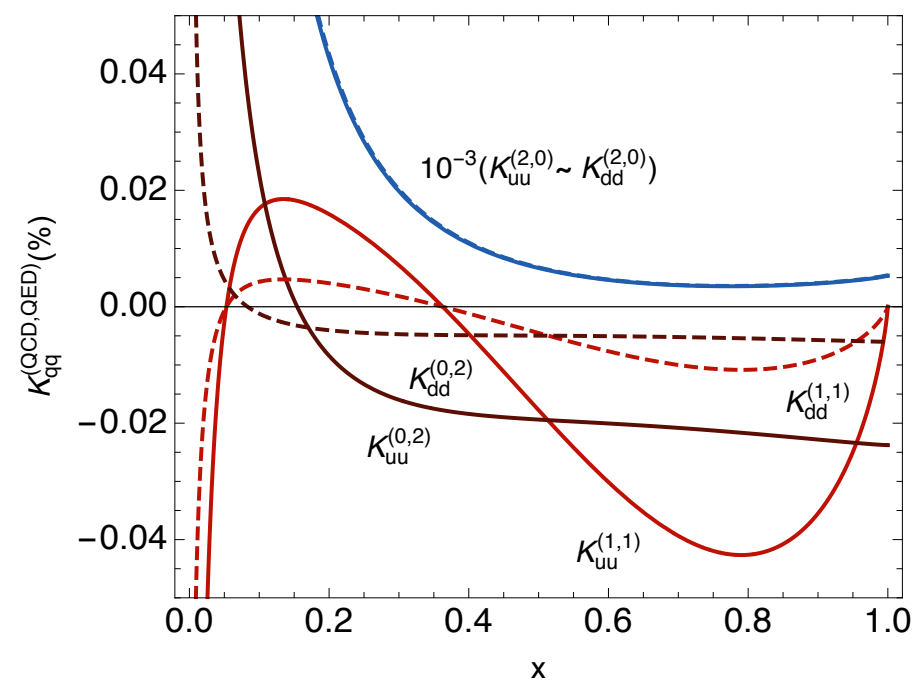

Figure 1. $K$ factors for the $q q$ splitting functions (\%). We separate among $u$ (solid lines) and $d$ (dashed lines) quarks to study the EM charge effects, and also among the different perturbative orders. Notice that $\alpha_{\mathrm{S}}^{2}$ terms are dominant (they are suppressed by a factor $10^{3}$ in this plot) and they exhibit almost the same behaviour for both $u$ and $d$ quarks.

and we notice that they share the same functional dependence, with the exception of the global normalization (influenced by the average over the quantum numbers of the initial particle). Finally, for the photon splitting kernel we have

$$
\begin{aligned}
P_{\gamma \gamma}^{(0,2)}=\left(\sum_{f} e_{f}^{4}\right) & {\left[-16+8 x+\frac{20}{3} x^{2}+\frac{4}{3 x}-(6+10 x) \ln (x)\right.} \\
& \left.-2(1+x) \ln ^{2}(x)-\delta(1-x)\right],
\end{aligned}
$$

that, at this order, includes both real and virtual corrections, in contrast with $\mathcal{O}\left(\alpha \alpha_{\mathrm{S}}\right)$ contributions [18].

\section{Phenomenological impact of QED corrections}

According to the expansion shown in eq. (2.6), the weight of higher-order corrections is suppressed by powers of $\alpha$ and $\alpha_{\mathrm{S}}$. In fact, working at $\mu=M_{Z}$, we have $a=1.2434 \times 10^{-3}$ and $a_{\mathrm{S}}=1.8860 \times 10^{-2}$. Thus, we anticipate that QED contributions to the AP kernels are small compared to pure QCD kernels. However, it might still happen that their effects become magnified due to the specific shape of the different PDFs. For this reason, we perform a study of the QCD and QED contributions to the splitting kernels to anticipate the possible consequences in the evolution of the PDFs.

Let's start with the analysis of the pure quark kernels $P_{q q}$. We define the ratio

$$
K_{a b}^{(i, j)}=a_{\mathrm{S}}^{i} a^{j} \frac{P_{a b}^{(i, j)}(x)}{P_{a b}^{\mathrm{LO}}(x)},
$$



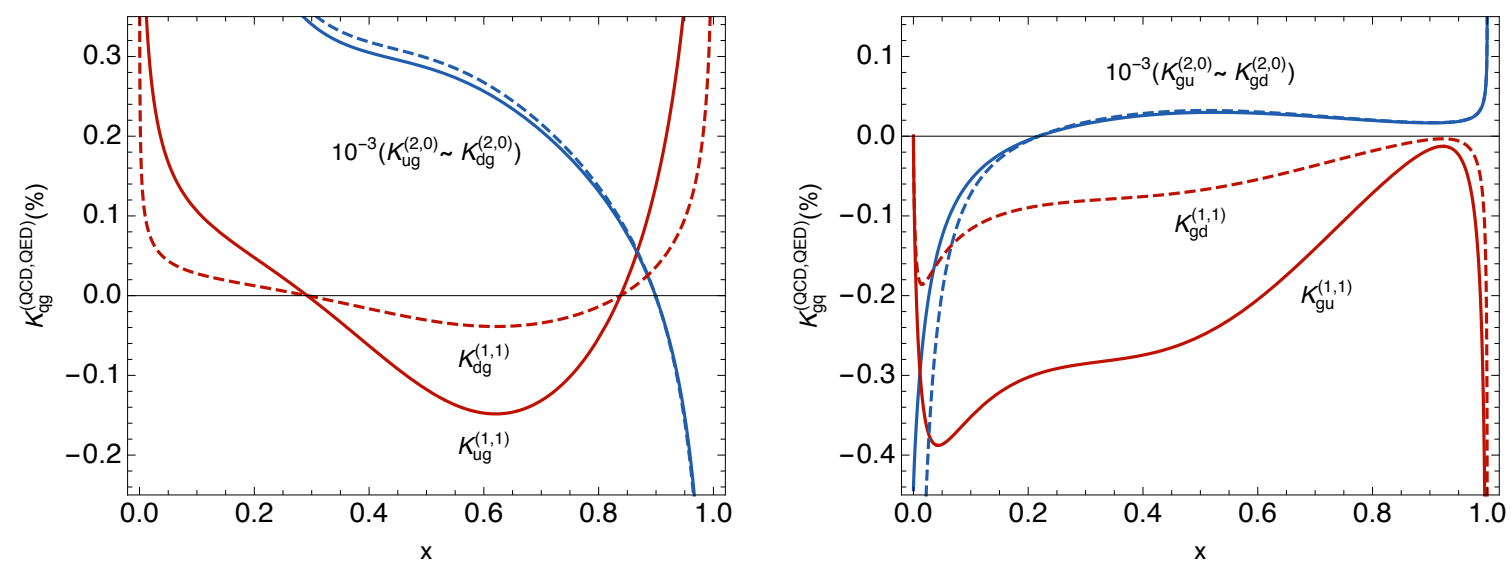

Figure 2. $K$ factors for the $q g$ (left) and $g q$ (right) splitting functions (\%). We include $\mathcal{O}\left(\alpha_{\mathrm{S}}^{2}\right)$ and $\mathcal{O}\left(\alpha \alpha_{\mathrm{S}}\right)$ contributions, and we also distinguish according to the EM charge of the involved quark; solid lines are used for $u$ quarks, whilst $d$ quarks are displayed with dashed lines. The $\mathcal{O}\left(\alpha_{\mathrm{S}}^{2}\right)$ term is dominant, and we suppress it by a factor $10^{3}$ in order to improve the graphical presentation.

where $P_{a b}^{\mathrm{LO}}(x)$ is the contribution to the evolution kernel at the lowest order in $\alpha$ and $\alpha_{\mathrm{S}}$. Notice that

$$
P_{a b}^{\mathrm{LO}}=a_{\mathrm{S}} P_{a b}^{(1,0)}+a P_{a b}^{(0,1)}
$$

i.e. $P_{a b}^{\mathrm{LO}}$ is not necessarily the lowest order contribution in only one of the couplings. We extract the $\mathcal{O}\left(\alpha_{\mathrm{S}}^{2}\right)$ contributions from refs. [27, 28] and the $\mathcal{O}\left(\alpha \alpha_{\mathrm{S}}\right)$ ones from ref. [18]; the resulting plot is given in figure 1. We distinguish there among quarks belonging to the up and down sector, respectively. As expected, deviations arising from QED corrections for $u$ quarks turn out to be bigger than those for $d$ quarks, since they are proportional to $e_{q}^{2} . P_{q q}^{(2,0)}$ terms are dominant in both cases; they represent a $\mathcal{O}(10 \%)$ correction, at least. However, the other corrections are of the same order of magnitude; approximately $\pm 0.04 \%$. Except from the singular behaviour in the limit $x \rightarrow 0$, there is a positive enhancement of $P_{q q}^{(1,1)}$ for $x \approx 0.10-0.15$ and a negative one for $x \approx 0.75-0.80$.

A similar analysis can be performed for $P_{q g}$ and $P_{g q}$ (figure 2). Pure QCD contributions to the splittings involving gluons are dominant against mixed QCD-QED ones; in any case, these contributions become increasingly relevant in the low $x$ region. Since gluon PDFs are magnified in that region, we expect a non-negligible effect in the evolution. The small EM charge separation observed in the $P_{q g}$ kernel for $\alpha^{2}$ correction originates from the normalization of the $K$ factor via eq. (4.2).

On the other hand, kernels involving a photon receive larger QED corrections, as observed for $P_{q \gamma}$ and $P_{\gamma q}$ (figure 3). Mixed $\mathcal{O}\left(\alpha \alpha_{\mathrm{S}}\right)$ QCD-QED contributions can reach the $20 \%$ level for $P_{q \gamma}$, while the two-loop QED terms modify the photon initiated kernel by up to $2 \%$ at small $x$. Furthermore, kernels involving leptons provide a non-trivial modification of QCD PDFs at $\mathcal{O}\left(\alpha^{2}\right)$. In figure 4 we plot the $K$ factors for $P_{l l}, P_{l \gamma}$ and $P_{\gamma l}$, respectively. Again, corrections reach the $2 \%$ level for the photon initiated kernels that can produce non-negligible effects to the photon distribution in a global analysis. 

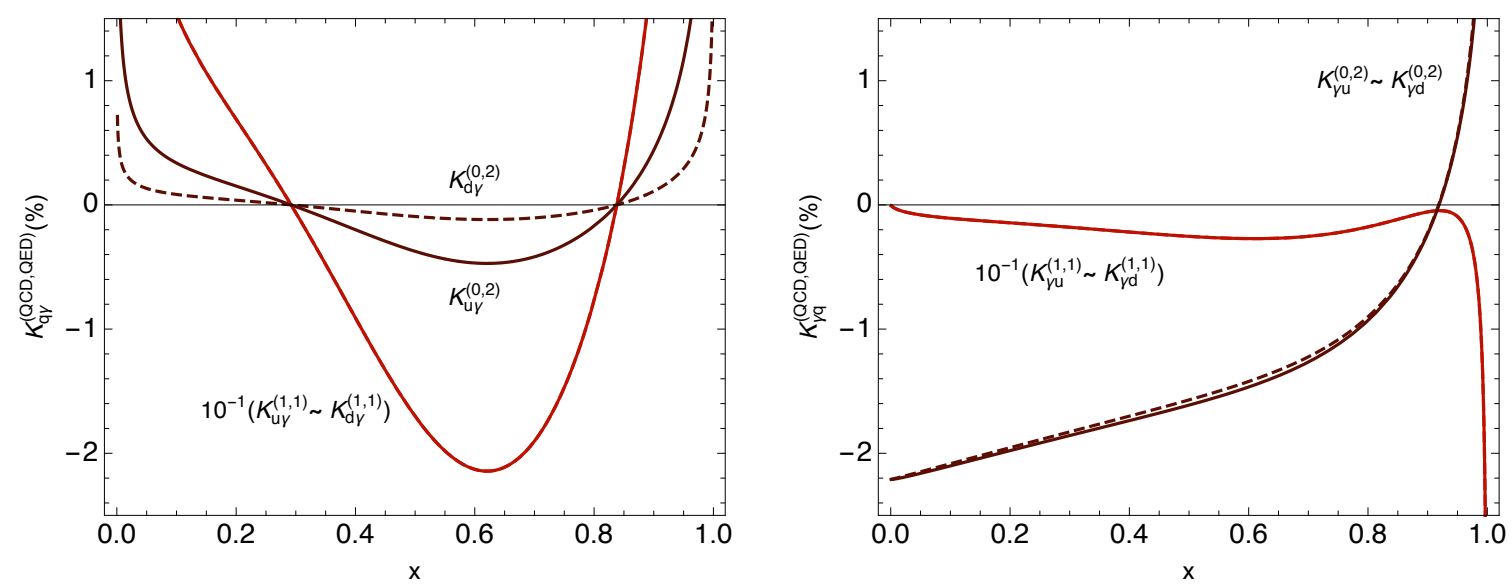

Figure 3. $K$ factors for the $q \gamma$ (left) and $\gamma q$ (right) splitting functions (\%). $\alpha^{2}$ and $\alpha \alpha_{\mathrm{S}}$ terms are included, with the last one being the dominant contribution. The EM charge distinction is enhanced in $P_{q \gamma}^{(i, j)}$ splitting, around $x \approx 0.65$. Mixed QCD-QED contributions are suppresed by a factor 10 to improve the visibility in the plot.

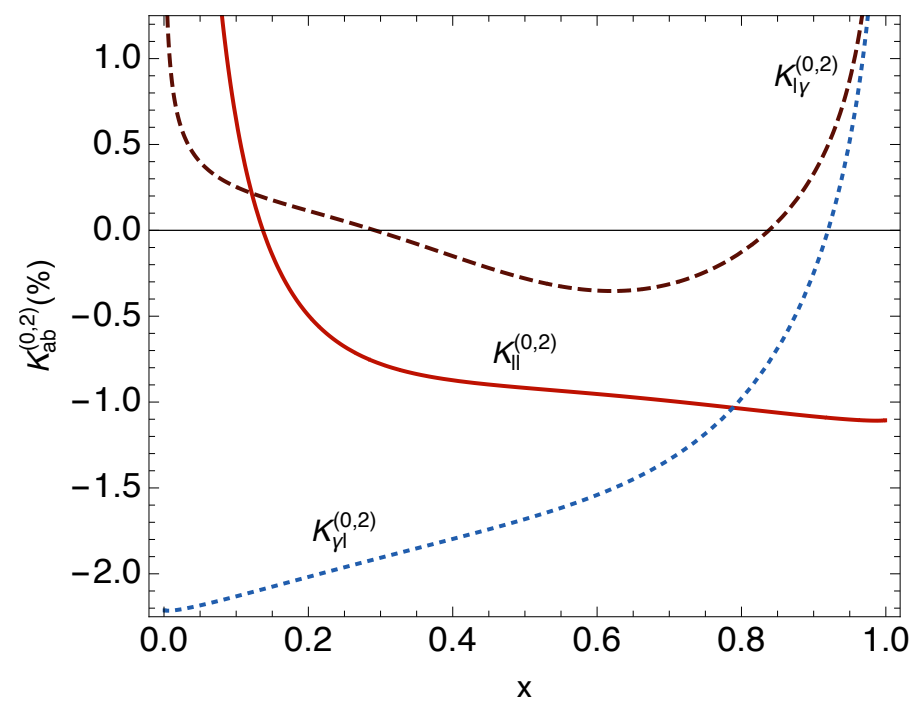

Figure 4. $K$ factors for the $\mathcal{O}\left(\alpha^{2}\right)$ corrections to the splitting functions involving leptons. In the range $0.1 \leq x \leq 0.95$, these contributions represent less than $2 \%$; they become more sizable near $x=0\left(\right.$ for $K_{l \gamma}^{(0,2)}$ and $K_{l l}^{(0,2)}$ ) and $x=1$ (for $K_{l \gamma}^{(0,2)}$ and $K_{\gamma l}^{(0,2)}$ ).

\section{Conclusions}

In this paper, we have presented for the first time explicit expressions for the Altarelli-Parisi splitting kernels to $\mathcal{O}\left(\alpha^{2}\right)$, completing the computation of the two-loop kernels needed to study the evolution of parton distributions to the precision achievable at the LHC. The full set of kernels includes those related to both photon and leptonic densities, the latest being allowed to mix in the evolution with parton distributions, mixing that starts at two-loops in QED. 

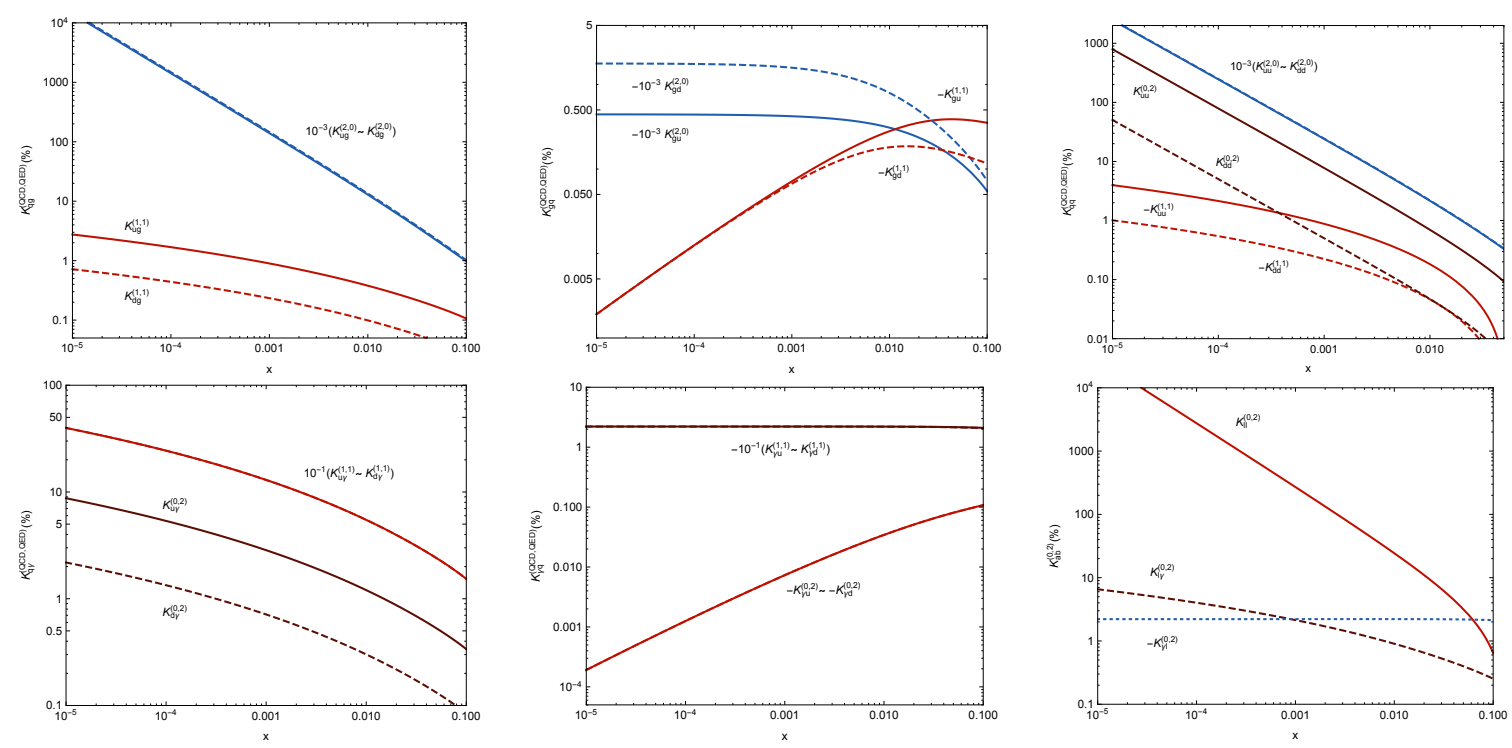

Figure 5. $K$ factors in the low- $x$ region. We plot the $\mathcal{O}\left(\alpha_{\mathrm{S}}^{2}\right), \mathcal{O}\left(\alpha \alpha_{\mathrm{S}}\right)$ and $\mathcal{O}\left(\alpha^{2}\right)$ corrections to the mixed QCD-QED splitting functions. In the first line, we considered $K_{q g}$ (left), $K_{g q}$ (center) and $K_{q q}$ (right). In the second one, we show $K_{q \gamma}$ (left), $K_{\gamma q}$ (center) and the $\mathcal{O}\left(\alpha^{2}\right)$ corrections to the kernels involving leptons (right).

We have obtained the corresponding kernels from the well-known NLO QCD corrections to the splitting functions, after carefully applying a well-defined algorithm to take the Abelian limit of the pure QCD expressions.

Finally, we have performed a phenomenological analysis to study the implications of these corrections in the splitting functions. We find that two-loop corrections are negligible for the pure quark kernels, but become sizable for $P_{q g}$ and $P_{g q}$ at small $x$ values (see figure 5). The effect of QED corrections turns out to generate $\mathcal{O}(2 \%)$ corrections for the splitting functions initiated by photons, which will alter the shape and size of the photon and leptonic distribution functions in a global analysis.

\section{Acknowledgments}

This work is partially supported by CONICET, ANPCyT, by the Spanish Government and EU ERDF funds (grants FPA2014-53631-C2-1-P and SEV-2014-0398) and by GV (PROMETEUII/2013/007).

Open Access. This article is distributed under the terms of the Creative Commons Attribution License (CC-BY 4.0), which permits any use, distribution and reproduction in any medium, provided the original author(s) and source are credited.

\section{References}

[1] S. Moch, J.A.M. Vermaseren and A. Vogt, Next-to-next-to leading order QCD corrections to the photon's parton structure, Nucl. Phys. B 621 (2002) 413 [hep-ph/0110331] [INSPIRE]. 
[2] S. Moch, J.A.M. Vermaseren and A. Vogt, The three loop splitting functions in QCD: the nonsinglet case, Nucl. Phys. B 688 (2004) 101 [hep-ph/0403192] [INSPIRE].

[3] A. Vogt, S. Moch and J.A.M. Vermaseren, The three-loop splitting functions in QCD: the singlet case, Nucl. Phys. B 691 (2004) 129 [hep-ph/0404111] [INSPIRE].

[4] A. Vogt, S. Moch and J. Vermaseren, Photon-parton splitting functions at the next-to-next-to-leading order of QCD, Acta Phys. Polon. B 37 (2006) 683 [hep-ph/0511112] [INSPIRE].

[5] S. Alekhin, J. Blumlein and S. Moch, The ABM parton distributions tuned to LHC data, Phys. Rev. D 89 (2014) 054028 [arXiv: 1310.3059] [INSPIRE].

[6] S. Dulat et al., New parton distribution functions from a global analysis of quantum chromodynamics, Phys. Rev. D 93 (2016) 033006 [arXiv: 1506.07443] [INSPIRE].

[7] P. Jimenez-Delgado and E. Reya, Delineating parton distributions and the strong coupling, Phys. Rev. D 89 (2014) 074049 [arXiv:1403.1852] [INSPIRE].

[8] ZEUS and H1 collaborations, H. Abramowicz et al., Combination of measurements of inclusive deep inelastic $e^{ \pm} p$ scattering cross sections and QCD analysis of HERA data, Eur. Phys. J. C 75 (2015) 580 [arXiv:1506.06042] [INSPIRE].

[9] L.A. Harland-Lang, A.D. Martin, P. Motylinski and R.S. Thorne, Parton distributions in the LHC era: MMHT 2014 PDFs, Eur. Phys. J. C 75 (2015) 204 [arXiv: 1412.3989] [INSPIRE].

[10] NNPDF collaboration, R.D. Ball et al., Parton distributions for the LHC run II, JHEP 04 (2015) 040 [arXiv: 1410.8849] [INSPIRE].

[11] A.D. Martin, R.G. Roberts, W.J. Stirling and R.S. Thorne, Parton distributions incorporating QED contributions, Eur. Phys. J. C 39 (2005) 155 [hep-ph/0411040] [INSPIRE].

[12] NNPDF collaboration, R.D. Ball et al., Parton distributions with QED corrections, Nucl. Phys. B 877 (2013) 290 [arXiv: 1308.0598] [INSPIRE].

[13] V. Bertone, S. Carrazza and J. Rojo, APFEL: a PDF evolution library with QED corrections, Comput. Phys. Commun. 185 (2014) 1647 [arXiv:1310.1394] [InSPIRE].

[14] C. Schmidt, J. Pumplin, D. Stump and C.-P. Yuan, CT14QED parton distribution functions from isolated photon production in deep inelastic scattering, Phys. Rev. D 93 (2016) 114015 [arXiv: 1509.02905] [INSPIRE].

[15] R. Sadykov, Impact of QED radiative corrections on parton distribution functions, arXiv: 1401.1133 [INSPIRE].

[16] S. Carrazza, Parton distribution functions with QED corrections, arXiv:1509.00209 [INSPIRE].

[17] V. Bertone, S. Carrazza, D. Pagani and M. Zaro, On the impact of lepton PDFs, JHEP 11 (2015) 194 [arXiv: 1508.07002] [INSPIRE].

[18] D. de Florian, G.F.R. Sborlini and G. Rodrigo, QED corrections to the Altarelli-Parisi splitting functions, Eur. Phys. J. C 76 (2016) 282 [arXiv:1512.00612] [INSPIRE].

[19] M. Gluck and E. Reya, Boundary conditions for the photon structure function in the leading and subleading order, Phys. Rev. D 28 (1983) 2749 [INSPIRE].

[20] M. Gluck, E. Reya and A. Vogt, Parton structure of the photon beyond the leading order, Phys. Rev. D 45 (1992) 3986 [INSPIRE]. 
[21] M. Fontannaz and E. Pilon, Reexamination of the photon structure function beyond the leading logarithm, Phys. Rev. D 45 (1992) 382 [INSPIRE].

[22] G.F.R. Sborlini, D. de Florian and G. Rodrigo, Double collinear splitting amplitudes at next-to-leading order, JHEP 01 (2014) 018 [arXiv:1310.6841] [INSPIRE].

[23] G.F.R. Sborlini, NLO QCD corrections to triple collinear splitting functions, arXiv: 1410.1680 [INSPIRE].

[24] G.F.R. Sborlini, D. de Florian and G. Rodrigo, Triple collinear splitting functions at NLO for scattering processes with photons, JHEP 10 (2014) 161 [arXiv:1408.4821] [INSPIRE].

[25] G.F.R. Sborlini, D. de Florian and G. Rodrigo, Polarized triple-collinear splitting functions at NLO for processes with photons, JHEP 03 (2015) 021 [arXiv: 1409.6137] [INSPIRE].

[26] G. Altarelli and G. Parisi, Asymptotic freedom in parton language, Nucl. Phys. B 126 (1977) 298 [INSPIRE].

[27] G. Curci, W. Furmanski and R. Petronzio, Evolution of parton densities beyond leading order: the nonsinglet case, Nucl. Phys. B 175 (1980) 27 [INSPIRE].

[28] W. Furmanski and R. Petronzio, Singlet parton densities beyond leading order, Phys. Lett. B 97 (1980) 437 [INSPIRE].

[29] R.K. Ellis and W. Vogelsang, The evolution of parton distributions beyond leading order: the singlet case, hep-ph/9602356 [INSPIRE].

[30] M. Roth and S. Weinzierl, QED corrections to the evolution of parton distributions, Phys. Lett. B 590 (2004) 190 [hep-ph/0403200] [INSPIRE].

[31] J. Blumlein, The theory of deeply inelastic scattering, Prog. Part. Nucl. Phys. 69 (2013) 28 [arXiv: 1208.6087] [INSPIRE].

[32] A.L. Kataev, Higher order $O\left(\alpha^{2}\right)$ and $O\left(\alpha \alpha_{s}\right)$ corrections to $\sigma_{\text {tot }}\left(e^{+} e^{-} \rightarrow\right.$ hadrons) and $Z$ boson decay rate, Phys. Lett. B 287 (1992) 209 [INSPIRE].

[33] J. Blümlein and S. Kurth, Harmonic sums and Mellin transforms up to two loop order, Phys. Rev. D 60 (1999) 014018 [hep-ph/9810241] [INSPIRE]. 